\title{
Trajectory Design and System Feasibility Analysis for Jovian Trojan Asteroid Exploration Mission Using Solar Power Sail
}

\author{
By Ryu FUnASE ${ }^{1)}$, Osamu MORI ${ }^{2)}$, Yoji SHIRASAwA ${ }^{2)}$ and Hajime YANO ${ }^{2)}$ \\ ${ }^{1)}$ Department of Aeronautics and Astronautics, The University of Tokyo, Tokyo, Japan \\ ${ }^{2)}$ Japan Aerospace Exploration Agency, Sagamihara, Japan
}

(Received June 24th, 2013)

\begin{abstract}
The solar power sail is a deep space probe that will be powered by a hybrid propulsion system with solar photon acceleration and ion engines to explore the outer planetary regions of the Solar System without having to rely on nuclear power. The Japan Aerospace Exploration Agency (JAXA) launched the world's first solar sail demonstration spacecraft, "IKAROS" (Interplanetary Kite-craft Accelerated by Radiation Of the Sun), in 2010. This spacecraft successfully demonstrated several key technologies related to the use of a solar power sail in a deep space flight environment. JAXA is currently planning an outer Solar System exploration mission using the demonstrated solar power sail technology, where the spacecraft will fly to Jupiter and perform a swing-by for a Jovian Trojan asteroid. This study undertook a trajectory design and system feasibility analysis for this mission. Candidate target asteroids were selected based on ballistic trajectory analysis, and then, electric-propulsion, continuous-thrust trajectory design was conducted to verify the conditions assumed for the ballistic analysis (e.g., estimate of steering loss due to the low-thrust trajectory). It was found that out of over 4000 Trojan asteroids, only 7 are feasible candidates considering the preliminary system design results. To broaden the choice of target asteroids, it will be necessary to reduce the weight of the solar power sail itself, its deployment mechanism, and the bus electronics.
\end{abstract}

Key Words: Solar Power Sail, Electric Propulsion, Low-Thrust Trajectory, Trojan Asteroid

\section{Introduction}

A solar sail, which consists of a huge, thin membrane that is deployed in space to generate a propulsive force by reflecting sunlight, is expected to be an essential propulsion system for future deep space exploration. This is because solar photon propulsion does not require any consumable fuel, and therefore allows further and longer space travel than conventional propulsion systems for which the velocity increment limit is determined by the amount of fuel that can be carried.

JAXA has proposed the concept of a "solar power sail" for future deep space exploration. This expands upon the concept of the solar sail. ${ }^{1)}$ A solar power sail combines a solar sail (photon propulsion) with additional electric power generation by using flexible solar cells attached to the sail membrane. The solar power sail spacecraft is thus powered by a hybrid propulsion system with solar photon acceleration and highly efficient ion engines driven by electric energy from the large flexible solar cells. This, in turn, provides a flexible and efficient orbital-control capability, even in the outer planetary regions of the Solar System, and does not have to rely on nuclear technology.

We have been studying and developing solar power sail technology for around 10 years with a goal of attaining advanced, original technologies for exploring the outer Solar System. Since 2002, we have conducted several experiments

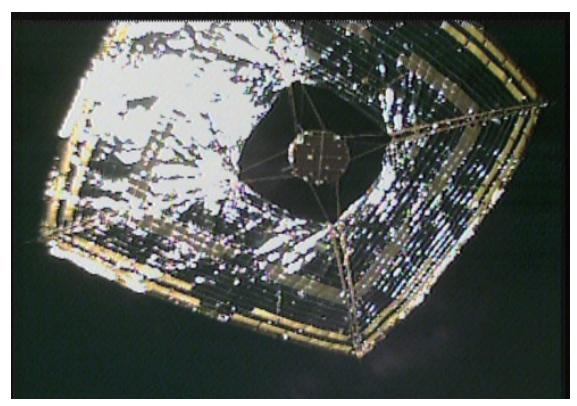

Fig. 1. IKAROS with fully extended sail viewed from a small detached camera probe in space.

on the ground using a vacuum chamber, a balloon, and a sounding rocket. After the completion of these experiments, we successfully launched the world's first solar sail demonstration spacecraft, called IKAROS (Interplanetary Kite-craft Accelerated by Radiation Of the Sun) in 2010 (Fig. 1). IKAROS was a precursor mission to demonstrate the key technologies for the solar power sail concept, which are (1) the deployment of a large sail in space; (2) solar power generation using thin-film solar cells attached to the sail; (3) confirmation of the acceleration produced by solar irradiation striking the sail; and (4) the interplanetary guidance, navigation, and control of the solar sail spacecraft. IKAROS successfully demonstrated these technologies and thus became the world's first solar sail. ${ }^{2)}$ 

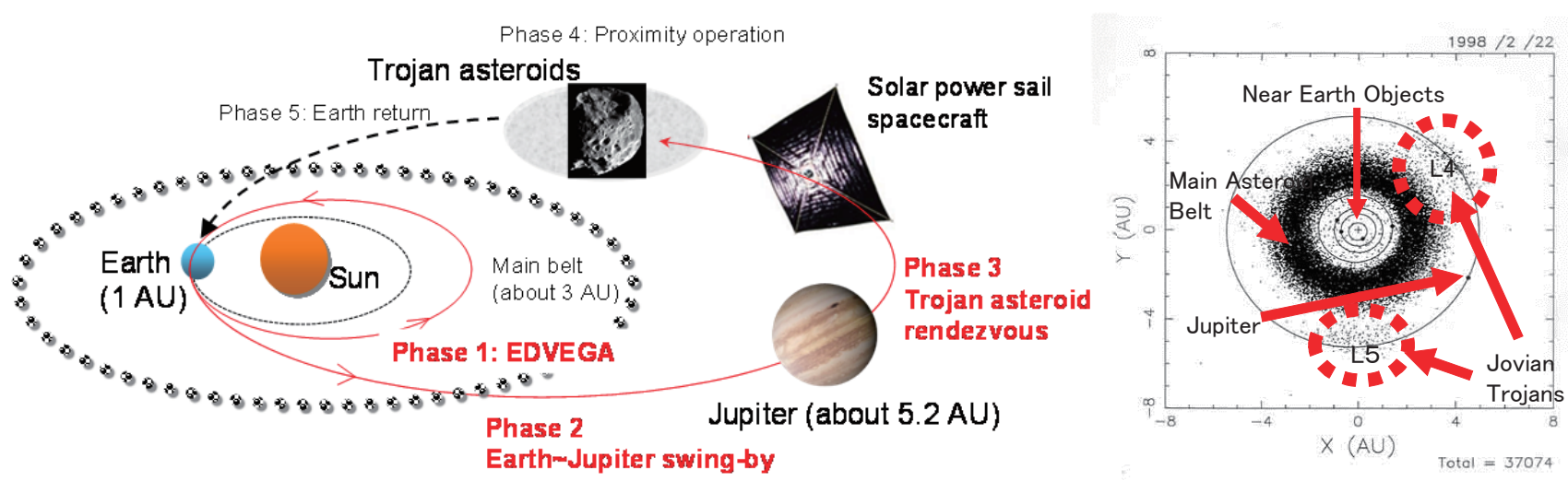

Fig. 2. Overview of solar power sail mission sequence (left) and position of Jovian Trojan asteroids at Lagrange points L4/L5 associated with the Sun-Jupiter system (right).

We are currently studying a mission for exploring the outer Solar System that will use a solar power sail and the electric propulsion technology demonstrated by IKAROS and the asteroid sample return spacecraft Hayabusa. ${ }^{3)}$ The goal of this mission is to fly to Jupiter, where the spacecraft will perform a swing-by for its destination, a Jovian Trojan asteroid. After rendezvousing with the asteroid, a sample return mission is optionally planned where the spacecraft will capture a sample of the asteroid and then bring it back to Earth. ${ }^{4)}$ Trojan asteroids are located at Lagrange points L4 and L5 associated with the Sun-Jupiter system (Fig. 2). JAXA plans to initiate the project in a few years and expects the launch to be in around 2020.

Previous research ${ }^{5)}$ explored ballistic trajectories (a direct transfer trajectory to an asteroid as well as a trajectory using gravity assists of Mars and Jupiter) and derived the generally easy-to-access Trojan asteroids, where the authors did not assume any spacecraft configuration or launch rocket. Our study focuses more on the trajectory design for the above mission using a solar power sail. The goal of this study is to design feasible trajectories to the Trojan asteroid considering the results of the preliminary solar power sail spacecraft system design as well as the launch rocket and launch window constraints, and thus to derive candidate target asteroids. In Section 2, we provide an overview of the mission, some of the new technologies to be demonstrated in this mission, and other scientific objectives. In Section 3, we perform a trajectory design and system feasibility analysis, focusing on the mission up until the rendezvous with the asteroid. In Section 4, we present the conclusions of this study.

\section{Jupiter and Trojan Asteroid Exploration Using Solar Power Sail}

\subsection{Mission overview}

The current scenario for the Jovian Trojan asteroid exploration mission consists of five phases. The first phase is an Electric Delta-V Earth Gravity Assist (EDVEGA) ${ }^{6}$ phase, whereby the departure velocity relative to Earth is efficiently increased. The second phase is the transfer from Earth to Jupiter, followed by a Jupiter swing-by. The third phase is a rendezvous with one of the Trojan asteroids at Lagrange points L4 or L5 associated with the Sun-Jupiter system (Fig.
2). Electric propulsion is utilized to achieve a large $\Delta \mathrm{V}$ for the rendezvous. The large amounts of power needed for the electric propulsion system are supplied by the solar power sail. The fourth and fifth phases address the optional sample return mission, which will capture an asteroid sample and bring it back to Earth, respectively.

We expect the launch to be in around 2020, and the overall mission duration from the launch to Trojan asteroid rendezvous will be around 10 years. (This does not include the Earth return phase of the optional sample return mission.)

\subsection{Technology demonstration missions}

This mission will demonstrate the following new technologies, which will be required for future exploration of the Solar System:

- fabrication of a large-membrane space structure and its deployment strategy

- hybrid propulsion using both photon acceleration and ultra-high-performance ion engines

- ultralight thin-film solar cells

- reaction control system (RCS) that functions at very low temperatures

- fuel cells integrated with the propulsion system

- EDVEGA technique using a hybrid propulsion system

- ultra-stable oscillator for one-way range or very long baseline interferometry (VLBI) precise orbit determination

- Ka-band communication for interplanetary missions Utilizing the large amounts of electric power provided by the large, ultralight thin-film solar cells, the spacecraft will drive its ultra-high $\mathrm{I}_{\mathrm{sp}}$ ion engines, even at Jovian distances ( $\left.>5 \mathrm{AU}\right)$, to rendezvous with a Trojan asteroid. The intended $I_{\mathrm{sp}}$ of the ion engines will be 6000 to $10000 \mathrm{~s}$, which is almost up to 3.3 times as efficient as existing ion engines. ${ }^{3)}$ A very large solar power sail will be deployed and extended to drive the ion engines. The area of the sail will be over 10 times larger than that of IKAROS. The goal of the EDVEGA technique, which utilizes this highly efficient electric propulsion system, is to increase the payload mass for outer Solar System exploration. These technologies will enable flexible orbital maneuverability even in the outer planetary regions of the Solar System, without having to rely on nuclear technology.

The RCS that functions at very low temperatures (below $-40{ }^{\circ} \mathrm{C}$ ) will drastically reduce the required heater wattage in 
outer Solar System environments, where the solar radiation intensity is very low. The fuel cells integrated with the RCS will realize a power storage that is very light but with a high power density. These are key technologies for advancing the bus technologies used in outer Solar System exploration. composition of the asteroids far beyond the current snow line and the internal structures implied by binary system measurements. The solar power sail mission will make a rendezvous with, and closely observe, a Trojan asteroid and will thus help to answer the above questions.
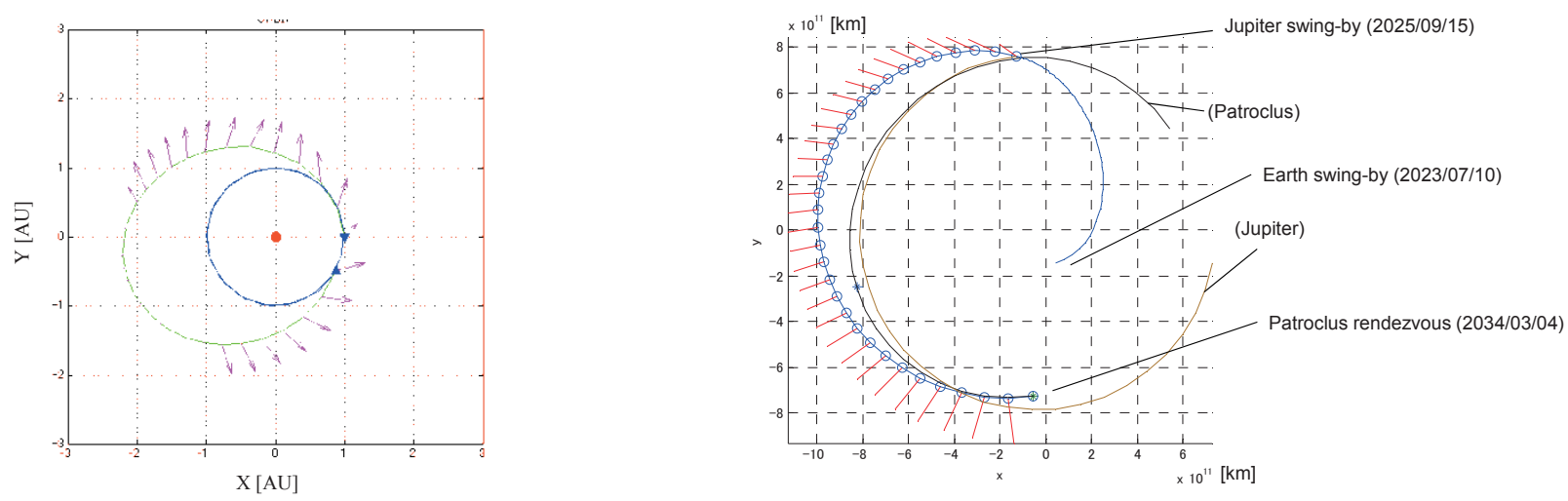

Fig. 3. Example of trajectory design. Two-rev EDVEGA trajectory in inertial coordinate (left), and transfer trajectory to Trojan asteroid Patroclus via a Jupiter swing-by (right). An arrow or a bar in the figure represents the thrust vector at each node of the trajectory.

\subsection{Scientific missions}

In addition to demonstrating these new technologies, other new and innovative science will be carried out by this spacecraft. ${ }^{7)}$

\section{Cruising science}

During the mission's long cruising period to the Trojan asteroid via the main asteroid belt and Jupiter, astronomical observations that are only possible in deep space will be performed. These will include (1) long baseline gamma ray burst detection and the polarimetry of highly energetic light sources, (2) infrared survey observation of the dark-age stars of the early universe in the whole sky beyond the foreground zodiacal light that originates primarily from the main asteroid belt, (3) zodiacal light measurement that will be directly compared with micrometeoroid impacts on the sail membrane of an area of up to $3000 \mathrm{~m}^{2}$, and (4) opportunistic flybys of near-Earth asteroids and main-belt asteroids, prior to reaching Jupiter and then its Trojan asteroids.

\section{Trojan asteroid exploration}

Jovian Trojan asteroids are one of the few remaining frontiers within our Solar System, and they may hold fundamental clues to the formation and evolution of the Solar System. The genesis of these asteroids is discussed by two competing hypotheses, namely, the classic model and the more recent Nice model. The former suggests that Trojan asteroids are mainly survivors of the building blocks of the Jupiter system, while the latter claims that they are intruders from outer regions of the Solar System that arrived after the planetary migration of the gas planets settled. In previous years, scientific investigations of these dark, distant asteroid reservoirs were largely dependent upon ground observations by large optical and spectroscopic telescopes, while few D-type asteroid analog meteorites were collected on Earth. However, thanks to recent development in observational technologies such as adaptive optics, statistical studies of the asteroids in the Jovian L4 and L5 regions have become possible. These studies have raised new questions about the

\section{Trajectory Design and System Feasibility Analysis}

In this section, trajectories to the Trojan asteroid are designed and the feasibility of the derived trajectories is analyzed considering the preliminary spacecraft system design results (such as the weight of the bus, the power generation capability of the solar power sail, and $\mathrm{I}_{\mathrm{sp}}$ of the ion engines). This section describes the derivation of candidate target asteroids.

\subsection{Analysis procedure}

Assumed trajectory sequence

We assumed a combination of 2-rev EDVEGA (two years of Earth-synchronous orbit with velocity increment by electric propulsion), a Jupiter transfer phase (ballistic trajectory), and a Trojan asteroid rendezvous phase (low-thrust trajectory) as the trajectory sequence leading up to Trojan asteroid rendezvous (Fig. 3). EDVEGA combines the $\mathrm{V}_{\infty}$-leveraging $\Delta$ V-EGA (Delta-V Earth Gravity Assist) technique ${ }^{8)}$ with a highly efficient electric propulsion system to increase the departure velocity relative to Earth. This technique enables the launch of a large payload to Jupiter, which would not be possible with a launch into a direct Jupiter transfer trajectory. A Venus-Earth-Earth Gravity Assist (VEEGA) trajectory with electric propulsion assistance would be possible, but an EDVEGA strategy was adopted because our preference was to keep the range of solar distance during the mission as small as possible. The range of solar distance with the EDVEGA trajectory is $>1 \mathrm{AU}$, whereas a VEEGA strategy would require a special thermal design for the spacecraft to enable it to survive the Venus solar distance (around 0.7 AU).

\section{Analysis conditions}

We assumed a launch by a Japanese H-IIA 204 (4S fairing) rocket with an ideal third-stage solid rocket kick motor. The value of $\mathrm{C} 3$ for the launch was assumed to be $28 \mathrm{~km}^{2} / \mathrm{s}^{2}$, which is required for the 2-rev EDVEGA trajectory. The launch capability under these conditions is about $2150 \mathrm{~kg}$. The 
Table 1. Feasible trajectory sequence and candidate target asteroids.

\begin{tabular}{|c|c|c|c|c|c|c|c|c|c|}
\hline Asteroid name & \begin{tabular}{|l|} 
Earth departure date \\
after EDVEGA \\
[yyyy/mm/dd]
\end{tabular} & $\begin{array}{l}\text { Asteroid arrival date } \\
{[\text { yyyy } / \mathrm{mm} / \mathrm{dd}]}\end{array}$ & $\begin{array}{l}\text { Vinf@asteroid } \\
{[\mathrm{km} / \mathrm{s}]}\end{array}$ & $\begin{array}{l}\text { Total DV estimate } \\
{[\mathrm{km} / \mathrm{s}]}\end{array}$ & $\begin{array}{l}\text { IES operation raito } \\
\text { (EDVEGA phase) }\end{array}$ & $\begin{array}{l}\text { IES operation raito } \\
\text { (Asteroid rendezvous phase) }\end{array}$ & $\begin{array}{l}\text { IES operating time } \\
{[\mathrm{h}]}\end{array}$ & \begin{tabular}{|l|} 
IES fuel \\
{$[\mathrm{kg}]$}
\end{tabular} & $\begin{array}{l}\text { Weight margin } \\
{[\mathrm{kg}]}\end{array}$ \\
\hline (2001 SD233) & $2025 / 09 / 24$ & $2036 / 05 / 07$ & 1.5 & 3.0 & 0.18 & 0.56 & 25932 & 69.6 & 273.4 \\
\hline (2005 GV192) & $2024 / 09 / 04$ & $2034 / 02 / 15$ & 1.3 & 2.7 & 0.18 & 0.49 & 22759 & 62.5 & 279.7 \\
\hline (2010 BJ70) & $2024 / 09 / 04$ & $2034 / 01 / 18$ & 0.9 & 2.1 & 0.19 & 0.35 & 16290 & 49.7 & 290.9 \\
\hline$(2010 \mathrm{GF} 8)=(2011$ PH11) & $2023 / 07 / 19$ & $2033 / 01 / 19$ & 1.7 & 3.5 & 0.23 & 0.65 & 30305 & 82.1 & 262.4 \\
\hline$(2010$ GF8) $=(2011$ PH11) & $2023 / 05 / 31$ & $2032 / 11 / 24$ & 1.8 & 4.7 & 0.47 & 0.68 & 33804 & 107.0 & 240.5 \\
\hline$(2010 \mathrm{GQ} 69)=(2012 \mathrm{TQ} 126)$ & $2025 / 10 / 01$ & $2035 / 04 / 11$ & 1.7 & 3.9 & 0.30 & 0.67 & 31318 & 89.1 & 256.3 \\
\hline$(2010 \mathrm{GQ} 69)=(2012 \mathrm{TQ} 126)$ & $2025 / 09 / 24$ & $2034 / 10 / 25$ & 1.5 & 4.6 & 0.55 & 0.61 & 30074 & 105.1 & 242.2 \\
\hline$(2010 \mathrm{GQ} 69)=(2012 \mathrm{TQ} 126)$ & $2024 / 09 / 04$ & $2034 / 09 / 27$ & 1.4 & 2.9 & 0.17 & 0.61 & 25038 & 66.8 & 275.9 \\
\hline$(2010 \mathrm{GQ69})=(2012 \mathrm{TQ} 126)$ & $2024 / 08 / 14$ & $2033 / 11 / 23$ & 1.4 & 3.2 & 0.28 & 0.67 & 25149 & 75.1 & 268.6 \\
\hline$(2010 \mathrm{GQ69})=(2012 \mathrm{TQ} 126)$ & $2024 / 08 / 07$ & $2035 / 07 / 04$ & 1.5 & 3.7 & 0.35 & 0.56 & 28116 & 86.6 & 258.5 \\
\hline$(2010$ GT147) $=(2006$ BK255) & $2024 / 09 / 04$ & $2034 / 04 / 12$ & 1.6 & 3.2 & 0.18 & 0.61 & 28071 & 73.9 & 269.7 \\
\hline (2010 LD60) & $2023 / 08 / 30$ & $2031 / 06 / 11$ & 1.5 & 4.6 & 0.55 & 0.65 & 30770 & 106.5 & 241.0 \\
\hline (2010 LD60) & $2023 / 08 / 09$ & $2032 / 08 / 04$ & 1.7 & 3.5 & 0.23 & 0.64 & 30025 & 81.8 & 262.7 \\
\hline (2010 LD60) & $2023 / 05 / 24$ & $2032 / 08 / 04$ & 1.7 & 4.7 & 0.49 & 0.64 & 33195 & 107.1 & 240.5 \\
\hline
\end{tabular}

Jupiter swing-by date was set to between Jan 1, 2025, and Dec 31,2029 , considering the likely launch timing (after 2021) and the duration of the EDVEGA phase (two years).

The effect of the solar radiation pressure on the sail was not considered in the trajectory calculation. This is because the solar radiation pressure applied to the solar power sail is essentially in the orbital radial direction (given that, for this mission, most of the sail area is covered by solar cells with a solar absorptance of almost 1) and the contribution of the radial acceleration to the orbital energy increment is not so large.

We considered all the Trojan asteroids that have been discovered to date as candidate target asteroids. Their orbital elements were obtained from the JPL Small-Body Database. ${ }^{9)}$ In this database, Jupiter Trojan asteroids are defined as asteroids trapped in Jupiter's L4/L5 Lagrange points (4.6 AU $<\mathrm{a}<5.5$ AU; e $<0.3$ ).

$\underline{\text { Step 1: Ballistic trajectory design }}$

Ballistic trajectories were designed for a sequence consisting of the Jupiter transfer phase, Jupiter swing-by, and Trojan asteroid rendezvous phase. Trajectories were globally searched using the "trajectory parts connection method."10) Ballistic trajectories for the Earth-to-Jupiter leg and the Jupiter-to-asteroid leg were calculated by solving Lambert problems. Feasible combinations of two legs were searched so that $\mathrm{V}_{\infty}$ at Jupiter and the Jupiter swing-by date for the two legs coincide with each other. To avoid the severe radiation environment around Jupiter, any feasible trajectory should have a swing-by radius at Jupiter of $>6 \mathrm{R}_{\mathrm{j}}$ (Jupiter radius, 7.15 $\left.\times 10^{4} \mathrm{~km}\right)$.

\section{Step 2: System feasibility analysis}

The approximate $\Delta \mathrm{V}$ for the EDVEGA phase and Trojan asteroid rendezvous phase was calculated for the derived ballistic trajectory sequences. Then, the spacecraft weight margin, ion engine operation ratio for each phase, and total operating time of the ion engine were calculated, and the constraints for these parameters were checked.

The $\Delta \mathrm{V}$ value for the EDVEGA phase was calculated considering the velocity difference between the launch C3 (5.3 $\mathrm{km} / \mathrm{s}$ ) and the Earth departure velocity for the Earth-to-Jupiter leg. $\Delta \mathrm{V}$ was calculated by dividing the velocity difference by a typical EDVEGA $V_{\infty}$-leveraging efficiency. We used the EDVEGA efficiency calculated by Hamasaki et al. ${ }^{11)}$ for this analysis. This study assumed a single-spin-type solar power sail with no solar radiation pressure acceleration applied, and the corresponding EDVEGA efficiency was used in our analysis.

The $\Delta \mathrm{V}$ value for the Trojan asteroid rendezvous phase was calculated from the relative velocity between the spacecraft and the asteroid when the spacecraft arrives at the asteroid. The overall $\Delta \mathrm{V}$ that should be produced by the ion thruster heads was calculated considering the steering loss for low-thrust trajectories and the cosine loss due to the angular difference between the ion thruster platform and the thrust axes of the ion thruster heads on the platform. We conservatively and empirically set these two figures to 1.4 and 0.95 .

The weight margin of the spacecraft was evaluated from the wet weight, dry weight, and the total $\Delta \mathrm{V}$ attained by the electric propulsion (as calculated above) and chemical propulsion $(200 \mathrm{~m} / \mathrm{s}$ for orbital correction and attitude control and $\mathrm{I}_{\mathrm{sp}}$ of $300 \mathrm{~s}$ ). Preliminary analysis suggested that the ion engine operation ratio constraint is the most limiting factor in obtaining feasible solutions. Therefore, to relax the requirement for the ion engine operation ratio, we determined (by trial and error) that the total weight of the spacecraft had to be as low as $1500 \mathrm{~kg}$ to be within the launch capability $(2150 \mathrm{~kg})$. This figure was determined so that we could secure a sufficiently large weight margin that would allow us to consider the anticipated additional resources required for the optional sample return mission $(200 \mathrm{~kg}$ ) and also obtain multiple feasible solutions. The dry weight of the spacecraft, which includes the weight of the bus electronics, the chemical and electric propulsion systems, the solar power sail and its deployment mechanism, and the scientific instruments, was set to $1098 \mathrm{~kg}$, based on our preliminary system configuration design results. The weight of the spacecraft structure, excluding the above components, was estimated to be $12 \%$ of the wet weight of the spacecraft $(1500 \mathrm{~kg})$ minus the weight of the propellant of the chemical and electric propulsion system. The weight of the propellant of the chemical and electric propulsion system for each designed trajectory was calculated from the wet weight and the value of $\Delta \mathrm{V}$.

The operating time of the ion engine was calculated for the EDVEGA phase and the Trojan asteroid rendezvous phase. The operating time can be calculated from the thrust of the ion engine. We assumed that the product of $I_{s p}[s]$ and the thrust/power ratio $[\mathrm{mN} / \mathrm{kW}]$ is constant $\left(9.0 \times 10^{4}\right.$ $[\mathrm{mN} \bullet \mathrm{s} / \mathrm{kW}])$ for an electron cyclotron resonance ion engine, ${ }^{3)}$ and the available thrust was calculated from the amount of 


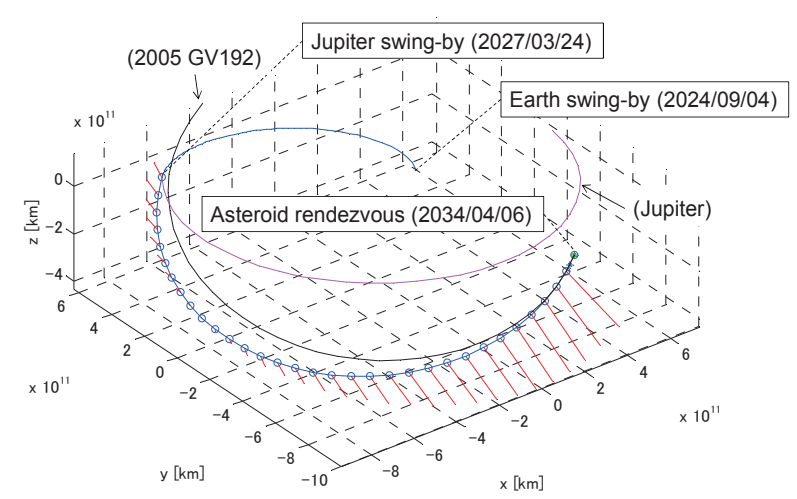

Fig. 4. Low-thrust trajectory design result for 2005 GV192.

power generated by the solar power sail at $5 \mathrm{AU}$ and the $\mathrm{I}_{\mathrm{sp}}$ of the ion engine system. The current design of the solar power sail assumes a sail area of $3000 \mathrm{~m}^{2}$. Therefore, the power generation capability at a solar distance of $5 \mathrm{AU}$ and a sun angle of $0^{\circ}$ was calculated to be $3.64 \mathrm{~kW}$ considering some system margins. The sun angle was assumed to be $45^{\circ}$ while the single-spin solar power sail spacecraft is using its ion engines, and we used this angle when calculating the power available for the ion engine operation. The " $\mu 10$ HIsp" $"$ ion engine system that is intended to be used for this mission is a higher- $I_{\mathrm{sp}}$ version of the " $\mu 10$ ",3) ion engine system used for the Hayabusa mission, and for this analysis, the $I_{s p}$ of the ion engines was determined to be $6000 \mathrm{~s}$, which is the lower limit of the range of $I_{s p}$ that can be achieved by modifying the current design of the $\mu 10 \mathrm{HIsp}$ (with an $\mathrm{I}_{\mathrm{sp}}$ of $10,000 \mathrm{~s}$ ). Preliminary analysis suggested that the ion engine operation ratio constraint is the most limiting factor in obtaining a feasible solution. For this reason, therefore, a lower $\mathrm{I}_{\mathrm{sp}}$ was chosen to achieve a larger thrust and relax the requirement for the ion engine operation ratio for the EDVEGA phase and the Trojan asteroid rendezvous phase.

The ion engine operation ratios for both the EDVEGA phase and the Trojan asteroid rendezvous phase were also calculated. A large operation ratio incurs the risk of a velocity increment shortage in the event of there being temporary operational troubles. Therefore, the operation ratio should not exceed a given limit, which we set to 0.7 for this study. This constraint was empirically derived for the operation ratio limit for one week (a 2-day margin for one week).

The total operating time of the ion engine should not exceed $40,000 \mathrm{~h}$, given the typical lifetime of a contemporary electron cyclotron resonance ion engine and expected developments in the technology.

Step 3: Low-thrust trajectory calculation

A low-thrust trajectory calculation was conducted to correspond to one of the selected (feasible) ballistic trajectory sequences. The objective of this calculation was to verify the conditions considered in the ballistic trajectory analysis (e.g., estimate of the steering loss for a low-thrust trajectory).

Direct collocation with nonlinear programming (DCNLP) was used as the trajectory optimization scheme, and the total $\Delta \mathrm{V}$ was used as the objective function to be minimized. We employed a nonlinear thrust inequality constraint that couples the thrust direction to the maximum thrust constraint. In this constraint model, the thrust direction determines the sail attitude, and the sail attitude determines the sun angle. Then, the available solar sail power and available thrust can be calculated. For the initial condition, the Jupiter departure date and the magnitude of the relative velocity with respect to Jupiter were fixed and the velocity direction was assumed to be free as long as it satisfies the Jupiter swing-by radius constraint. For the terminal condition, the spacecraft position and velocity should be identical to that of the target asteroid. The time of flight (TOF) was not constrained.

\subsection{Results}

A trajectory design and system feasibility analysis was conducted according to the above procedure. Table 1 lists the derived feasible trajectory sequences, and shows the feasible combinations of target asteroids and representative trajectories. The weight margin for each combination is also listed. Each combination satisfies the feasibility criteria (weight margin, operating time, and operation ratio of the ion engine).

Fig. 4 shows the results of the low-thrust calculation. This trajectory was calculated for the second trajectory sequence in Table 1. The value of $\Delta \mathrm{V}$ for this low-thrust trajectory was about $1.8 \mathrm{~km} / \mathrm{s}$, which was consistent with the ballistic $\Delta \mathrm{V}$ (about $1.3 \mathrm{~km} / \mathrm{s}$ ) and the estimated steering loss for the low-thrust trajectory (1.4). It can be said that the conditions assumed for the feasibility analysis were appropriate. However, low-thrust trajectory analyses for more of the cases listed in Table 1 will be required to statistically confirm that the assumed conditions are, in fact, appropriate.

It was found that, out of over 4000 Jupiter Trojan asteroids, only 7 are feasible. The most limiting factor in obtaining a feasible solution was the constraint on the ion engine operation ratio, and this is mainly because the dry weight of the spacecraft (which includes the weight of the solar power sail) is so large that the available acceleration is very limited such that only very low $\Delta \mathrm{V}$ trajectories are feasible considering this constraint on the ion engine operation ratio. In other words, the ion engine operating time tends to become large owing to the large weight of the spacecraft.

To broaden the feasibility of the mission and the choice of target asteroids, it will be necessary to reduce the weight of the solar power sail, its deployment mechanism, and the bus electronics.

Further investigation will also be required to determine which of the selected asteroids (Table 1) are of more scientific value in order to achieve the scientific goals described in Section 2.3.

\section{Conclusions}

In this study, we conducted a trajectory design and system feasibility analysis for a Jovian Trojan asteroid exploration mission using a solar power sail. This mission plans to fly to Jupiter, where the spacecraft will perform a swing-by for its destination, a Trojan asteroid.

The trajectory sequence from launch to arrival at the Trojan asteroid was designed based on ballistic analysis. Then, the feasibility of the derived trajectory sequences was analyzed in 
terms of the constraints of the spacecraft weight margin, total operating time, and operation ratio of the ion engine. Next, a low-thrust trajectory calculation was conducted for one of the selected feasible ballistic trajectory sequences in order to verify the conditions assumed for the ballistic trajectory feasibility analysis.

Our results showed that, out of over 4000 Jupiter Trojan asteroids, only 7 asteroids are feasible. This is mainly because the dry weight of the spacecraft (which includes that of the solar power sail) is so large that the available acceleration is very limited. To broaden the feasibility of the mission and the choice of target asteroids, it will be necessary to reduce the weight of the solar power sail, its deployment mechanism, and the bus electronics. JAXA plans to initiate this project in a few years and anticipates the launch in around 2020. Further studies and technology development will be conducted in support of this mission.

\section{References}

1) Kawaguchi, J.: A power sailer mission for a Jovian orbiter and Trojan asteroid flybys, COSPAR04-A-01655, Abstracts of 35th COSPAR Scientific Assembly, Paris, France, 18-25 July, 2004.

2) Tsuda, Y., Mori, O., Funase, R., Sawada, H., Yamamoto, T., Saiki, T., Endo, T., Yonekura, K., Hoshino, H. and Kawaguchi, J.: Achievement of IKAROS - Japanese Deep Space Solar Sail Demonstration Mission, Acta Astronautica, 82 (2013), pp.183-188.

3) Kuninaka, H., Nishiyama, K., Funaki, I., Yamada, T., Shimizu, Y. and Kawaguchi, J.: Powered Flight of Electron Cyclotron Resonance Ion Engines on Hayabusa Explorer, Journal of Propulsion and Power, 23 (2007), pp.544-551.
4) Matsumoto, J., Funase, R., Mori, O., Shirasawa, Y., Ono, G., Hamasaki, T., Hayashi, N., Chujo, T., Motooka, N. and Tanaka, K.: Mission Analysis of Sample Return from Jovian Trojan Asteroid by Solar Power Sail, 2013-k-57, 29th International Symposium on Space Technology and Science, Nagoya, Japan, 2013.

5) Simanjuntak, T., Nakamiya, M. and Kawakatsu, Y.: Mission Design for Jupiter Trojans Rendezvous Mission, Advances in the Astronautical Sciences, 136 (2010), AAS 10-246, pp.2169-2183.

6) Kawaguchi, J.: Solar Electric Propulsion Leverage: Electric Delta-Vega (EDVEGA) Scheme and its Application, Advances in the Astronautical Sciences, 108 (2001), AAS 01-213, pp.1577-1586.

7) Yano, H., Mori, O., Funase, R., Matsuura, S., Fujimoto, M., Takashima, T. and Solar Power Sail/Jovian Trojan Working Group: Solar Power Sail, the Jovian Trojan Explorer and Deep Space Astronomical Platform, 6251, Abstracts of Asteroids, Comets, Meteors (ACM) 2012, Niigata, Japan, May 2012.

8) Sims, J. A., Longuski, J. M. and Staugler, A. J.: $V_{\infty}$ Leveraging for Interplanetary Missions: Multiple-Revolution Orbit Techniques, Journal of Guidance, Control and Dynamics, 20 (1997), pp.409-415.

9) JPL Small-Body Database Search Engine, http:// http://ssd.jpl.nasa.gov/sbdb_query.cgi.

10) Kawakatsu, Y., Abe, M. and Kawaguchi, J.: Mission Analysis for Sample Retrieval from a Primitive Near-Earth Asteroid, Transactions of the Japan Society for Aeronautical and Space Sciences, Space Technology Japan, 7 (2009), pp.33-41.

11) Hamasaki, T., Funase, R. and Kawaguchi, J.: On the Efficiency of EDVEGA by Single Spin Type and Dual Spin Type Solar Power Sail, Proceedings of 22nd Workshop on JAXA Astrodynamics and Flight Mechanics, Sagamihara, Japan, pp.140-145, July 2012.

12) Hayashi, H., Kuninaka, H., Usui, M., Hosoda, S., Koizumi, H., Nakayama, Y., Shimizu, Y. and Nishiyama, K.: R\&D on $10,000 \mathrm{sec}$ Isp Ion Engine Driven by Microwave, AIAA 2008-4810, 44th AIAA/ASME/SAE/ASEE Joint Propulsion Conference \& Exhibit, Hartford, USA, 2008. 